\title{
STRAFEN UND TORTUREN DER TIBETER
}

\author{
(Beitrag zur tibetischen Rechtspflege)
}

\author{
Siegbert Hummel.
}

\section{EINFÜHRUNG}

Über tibetische Justiz und die von ihr angewendeten Strafen und Torturen finden sich in europäischen Reisewerken oder in Monographien zur Kulturgeschichte Tibets leider nur gelegentlich verstreute Hinweise. Davon sind wieder nur ganz wenige als Augenzeugenberichte der betreffenden Autoren bekannt. Da über die Verhältnisse im tibetischen Rechtsleben seit der kürzlich erfolgten Eingliederung Tibets in das chinesische Reich noch keine ausreichenden Nachrichten vorliegen und mit Wahrscheinlichkeit eine Angleichung an die heutige chinesische Rechtsprechung anzunehmen ist, müssen wir uns im folgenden auf die Zeit bis zum Einmarsch der chinesischen Truppen beschränken. Das gewährleistet zugleich ein Bild echter tibetischer Gepflogenheiten, die in Kürze der Geschichte angehören werden.

Kurz zusammengefaßt hat die wichtigsten Strafen in Westtibet A. CunningHAM. in seinem heute noch gültigen Werk über Ladak (La-dvags) ${ }^{1}$. Eine Sonderstellung nehmen zweifellos H. S. LANDors Erlebnisse ein ${ }^{2}$, da die von diesem Reisenden aus der Erinnerung gezeichneten Bilder der von ihm selbst erlittenen Folterungen trotz der oftmals lautgewordenen Skepsis durch die von mir hiermit veröffentlichten tibetischen Zeichnungen ${ }^{3}$ als richtig bestätigt werden. Eine vollständige Übersicht tibetischer Strafen im Bilde ist mir bisher nicht bekannt geworden. Insofern mögen unsere Zeichnungen ein besonderes Interesse verdienen. Sie sollen von einem Tibeter in Westtibet angefertigt worden sein und waren von S. H. RIBBACH, dem bekannten Herrnhuter Missionar, allem Anschein nach für eine Veröffentlichung bestimmt.

Die beigegebenen Beschriftungen in tibetischer Sprache bieten einige Merkwürdigkeiten, die für unsere Kenntnis der tibetischen Sprache nicht ohne Bedeutung sind. Ein Vergleich mit den einschlägigen Wörterbüchern ${ }^{4}$ könnte zunächst die Vermutung aufkommen lassen, daß es sich um orthographische Fehler handelt, die vielleicht aus der Niederschrift nach dem Diktat verständlich werden. Ich habe die zu erwartende Schreibweise hinter die Abweichungen in eckiger Klammer eingefügt. Die ziemlich ungeläufigen und in den Wörterbüchern zum größten Teil fehlenden Varianten sind aber offenbar phonetische Bildungen, denen eine gewisse Regelhaftigkeit zugrunde liegt, die auch schon B. LAUFER in dem von ihm herausgegebenen Sühngedicht der Bonpo, Wien 1900, aufgefallen sind. Auch dort haben wir es mit einer westtibetischen Arbeit zu tun. Für die von LAUfER versuchsweise skizzierten phonetischen Gesetze (1. c., S. $20 \mathrm{ff}$.) bieten somit die Beschriftungen unserer Bilder weiteres wertvolles Belegmaterial. So können wir folgende Regeln fixieren:

1. Präfigiertes $r$ vor ts, z. B. in brtson für das übliche btson (Bild 10), vgl. B. LAUFer, l. c., S. 21.

2. Abgeworfene Präfixe g und b, z. B. cig für gcig (14), tub für gtub (11), cug für bcug (3) und tum für btum oder als ungewöhnliche Perfektform von 'thum-pa (8 und 13), vgl. B. LAUfER, l. c., S. 21.

1 A. Cunningham, London 1854, S. $262 \mathrm{ff}$.

2 H. S. Landor, Auf verbotenen Wegen, 8. Aufl. Leipzig 1910, S. $395 \mathrm{ff}$.

3 Sammlung des Verfassers. Die Größe der Reproduktion der Tuschzeichnungen beträgt nahezu drei Viertel des Originals.

* A. H. Jäschke, A Tibetan-English Dictionary, London 1881. - Sarat Chandra Das, A Tibetan-English Dictionary, erweiterte Auflage, Calcutta 1951. 
3. Vertauschte Präfixe, z. B. mdon für 'don (9), rkyur für skyur (12), bcod für gcod (7) und bsal für gsal (16), vgl. B. LAUfer, 1. c., S. 21.

4. Abgeworfenes schließendes s, z. B. lcag für lcags $(4,6,12)$, vgl. Laufer, l. c., S. 22.

Die Abbildungen geben natürlich nur die körperlichen Strafen (tib.: Lus-chad) wieder. Daneben bestehen aber auch die sogenannten bTson-chad als Freiheitsstrafen oder als Verbannung im besonderen, ferner die Nor-chad, die Strafen am Eigentum, d. h. am beweglichen und unbeweglichen Vermögen bzw. Geld. Von der Verwandlung körperlicher Strafen in Geldstrafen berichtet schon A. Cunningham (l. c., S. 265). Wirksame und einschneidende Eingriffe in den tibetischen Strafvollzug hat bekanntlich der XIII. Dalai-Lama Ngag-dbang-blo-bzang-thub-ldan ( $\dagger 1933$ ) versucht. So wußte er in seinem Machtbereich die Verstümmelung weithin zu verhindern. Die den Grundsätzen des Buddhismus entgegenstehende Todesstrafe gestattete er nur noch in ganz vereinzelten, besonders schweren Fällen. Für viele Körperstrafen hat er gemeinnützige Arbeiten, z. B. Straßenbau oder Arbeit in den staatlichen Gärten, angeordnet ${ }^{5}$, womit er nach europäischer Auffassung moderne und humane Ideen in das sonst weithin grausame, an unsere mittelalterlichen Zustände erinnernde Rechtsleben der Tibeter einführte.

Interessant sind die Abweichungen sowohl im Strafmaß als auch im Charakter der Züchtigungen sowie in der Anwendung der Strafen auf die verschiedenen Delikte in den einzelnen Landesteilen, wobei sich die Praxis in Osttibet von der westtibetischen im allgemeinen durch größere Härte unterscheidet. Das wird jedoch aus dem unterschiedlichen kulturellen Zustand der verglichenen Gebiete verständlich. Aber selbst in ein und demselben Landesteil fallen noch Verschiedenheiten auf, wie aus A. TAfels Berichten 6 über die Bestrafung von Diebstahl oder gewaltsamem Raub in Osttibet hervorgeht ${ }^{7}$. An Stelle einer für das gesamte Land verbindlichen einheitlichen Regelung tritt bis in die Gegenwart die örtlich variierende und nur in ganz großen Zügen einheitliche Tradition, wobei einige der Strafen zweifellos chinesischen Ursprungs sind, so z. B. das Strafholz, tib.: Tshe-sgo, im allgemeinen als Kang bekannt ${ }^{8}$, oder das Durchstoßen des Ohres mit einem Pfeil ${ }^{9}$.

Um eine Übersicht in die auf unseren Zeichnungen dargestellten Strafen zu bringen, habe ich sie so geordnet, $\mathrm{da} ß$ von den nach unserem Empfinden weniger qualvollen Handlungen ausgegangen und zu den immer grausameren fortgeschritten wird. Das braucht jedoch nicht immer dem Rechtsempfinden des Tibeters zu entsprechen. So wird z. B. die Verstümmelung, insbesondere die Enthauptung, aus alten vorbuddhistischen und vulgären Jenseitsvorstellungen, wie sie auch noch in der lamaistischen Lehre vom Bar-do, dem vorübergehenden Schattendasein zwischen Tod und Neugeburt, enthalten sind ${ }^{10}$, besonders verabscheut. Der Schatten soll nicht mit den Zeichen der Verstümmelung geschändet werden. Über die tibetischen Vorstellungen von der Schwere der einzelnen Strafen könnte allein eine systematische Untersuchung der tibetischen Strafjustiz aufklären, eine Untersuchung, die umsomehr erwünscht ist, als die alte tibetische Rechtsprechung bald der Vergangenheit angehören wird.

Die Abbildungen 17, 18, 19 und 20 zeigen einige Foltern. Bei Bild 17 (Mig-thag-gtong-ba $=$ Augen an Schnüren) werden die Augenlider mit Schnüren und eisernen Häkchen an der Brust festgeheftet. Nach JäsCHKE (l. c., S. 414) war diese Folter in Zentraltibet heimisch. Die Streckfolter auf Nr. 18 (mDa'-sprad-'gal ['gel] -ba = In Bogenspannung aufhängen) ist uns in ähnlicher Form aus LaNDORS Bericht und Zeichnung (l. c., S. 433 f.) für das westliche Zentraltibet näher bekannt. Eine Verschärfung der Tortur durch das Aufträufeln von heißem Siegellack zeigt Bild 19 (Lathig-rgyag-pa $=$ Mit flüssigem Siegellack betropfen). Dabei bleibt der Delinquent ans Streckholz gefesselt (rGyang-shig-sprang-pa [brKyang-shing-sprad-pa]). Die zugehörige Abbildung zeigt oben

5 Vgl. Ch. Bell, Portret van de Dalai Lama, Amsterdam 1949, S. $146 \mathrm{ff}$.

6 A. TAfel, Meine Tibetreise, Stuttgart 1914, Bd. 2 S. 147.

${ }^{7}$ So urteilen schon die T'ang-Annalen (in : S. W. Bushel., The Early History of Tibet, JRAS Vol. XII, London 1880, S. 441).

8 Vgl. Bild 1 und 2.

9 Vgl. Bild 5.

10 Vgl. S. Hummel, Nichtanimistisches und Animistisches im Lamaismus (in : Jahrbuch des Museums für Völkerkunde zu Leipzig Bd. XII, Leipzig 1954). 
in Vergrößerung das für den flüssigen Siegellack verwendete Sieb. Weitere Torturen sind nach E. KalvaguCHI ${ }^{11}$ in Zentraltibet die unter die Fingernägel getriebenen scharfen Bambusspäne, eine Folter chinesischen Ursprungs, oder die auf den Kopf gesetzten und beliebig erhöhten Steinlasten. In Verbindung mit den Torturen erwähnt A. CunsinghaM (l. c., S. 266) auch die Praxis der sogen. Gottesgerichte in solchen Zweifelsfällen, wo Tatbeweise fehlen oder die Folter zu keinem Geständnis führt. So muß mit unverletzter Hand ein Stein aus kochendem Öl geholt werden, bisweilen liegen auch ein weißer und ein schwarzer Stein im Öl oder in kochender Milch, wobei dann der weiße Stein ergriffen werden muß, wenn die Unschuld völlig erwiesen sein soll. Ein anderes Gottesgericht besteht im Tragen eines glühenden Eisens in unverletzter Hand. Beide Gottesgerichte sind nach Cunvingham zentraltibetischen Ursprungs (vgl. hierzu auch M. Hermanss, Mythen und Mysterien der Tibeter, Köln 1956, S. 82 f.).

\section{Die Abbildungen}

Es handelt sich also im Folgenden nicht um willkürliche Grausamkeiten, wie sie z. B. in Kriegszeiten vorkommen, sondern um ordnungsgemäße Strafen, wie sie im tibetischen Rechtsleben üblich sind.

1. Tshe-sgo $=$ Strafholz (chinesisch: Chia ${ }^{12}$ ), sogenannter Kang ${ }^{13}$. Zwei runde Bretter, die mit einem Schloß verschlossen werden. Das runde Strafholz ist eine Art Pranger, der im allgemeinen bei geringfügigen Vergehen Anwendung findet ${ }^{14}$. Auf einem angehefteten Zettel stehen Schuld und Strafe zu lesen, d. h. die Anzahl der Tage oder auch der Jahre im Strafholz. Die Verurteilten können sich nicht selbst sättigen, sondern müssen ernährt werden, was meist durch die Verwandten geschieht. Nachts haben sie sich im Gefängnis einzufinden. Die gleiche Praxis gilt auch für die unter 2 genannten Sträflinge.

2. $s G o-g \cdot y \circ g-p a=D a s$ Strafholz anlegen, wörtlich: Tür anlegen. Die Strafhölzer sollen eine Ähnlichkeit mit einer Türfüllung haben. Sie werden wie der runde Kang verschlossen und mit einem Strafzettel versehen. Die Vierkantbretter sind schwerer als die runden und messen ca. $90 \mathrm{~cm}$ an den Seiten bei einer Stärke von $3 \mathrm{~cm}$. In leichteren Delikten darf das Vierkantbrett nachts gegen das runde eingetauscht werden. Mitunter ist mit der Buße im Kang noch körperliche Züchtigung oder Verbannung (gNas-na-dgar-ba) verbunden, letztere z. B. in Westtibet bei Mord oder in Zentraltibet bei politischen Vergehen. Von der Anwendung des schweren Kang im Anschluß an einen Attentatsversuch auf den XIII. Dalai-Lama und nach einer Revolte in Lha-sa im Jahre 1947 berichten E. Kawaguchi (1.c., S. 374 mit Abb. auf S. 378) und H. Harrer ${ }^{15}$. Dabei wurden mit schwerem Kang und anschließender Verbannung bzw. lebenslänglicher Haft in der Verbannung nur indirekt an der Verschwörung Beteiligte bestraft.

Bei der Verbannung wird nach A. Cunningham (1. c., S. 263) den Verurteilten in Westtibet mit einem glühenden Eisen ein Hundekopf in die Stirn gebrannt. Diese Sitte soll nach Cunningham in Kâschmîr bereits im 10. Jh. bestanden haben. Dieser Zeichnung liegt wohl die Vorstellung von der dämonischen Unterweltsbezogenheit des Hundes zugrunde, die aus iranischen Traditionen herzuleiten ist ${ }^{16}$. Aus den gleichen Motiven treibt man z. B. noch heute beim Neujahrsfest in Lha-sa den Sündenbock mit einem Hund in die Einsamkeit ${ }^{17}$.

11 E. Kawaguchi, Three Years in Tibet, Madras 1909, S. $376 \mathrm{f}$.

12 Vgl. W. RódenberG, Chines.-Deutsch. Wörterbuch, Hamburg 1936, Nr. 643.

13 portugiesisch : canga $=$ Joch.

14 Vgl. L. A. WadDELL, Lhasa and its Mysteries, London 1905, S. 339.

15 H. HARrer, Sieben Jahre in Tibet, Wien 1952, S. $188 \mathrm{f}$.

16 Vgl. S. Hummel, Der Hund des Daitschin-Tengri (in: Geogr. Helvetica, Zürich 1955, 3).

17 'Vgl. R. DE NebEsky-Wojkowitz, Oracles and Demons of Tibet, s'Gravenhage 1956, S. $507 \mathrm{f}$. Zur Unterweltsbezogenheit des Sündenbockes vgl. A. Jeremias, Das Alte Testament im Lichte des Orients, 4. Aufl., Leipzig 1930, S. 442. - Zur Dämonie des Hundes vgl. E. Unger, Der Hund im Dämonenglauben des Altertums und des Mittelalters (in: Der Terrier, Frankfurt a. M. 1954, S. $241 \mathrm{f}$.). 
3. Shing-gong-an-ltos [gdos]-la-cug [bcug]-pa = In den Stock legen. Mit dieser ursprünglich chinesischen Strafe werden Einbrecher und Diebe bestraft.

5. rKang-lcag [lcags]-lag-lcag [lcags] $=F u \beta$ - und Handeisen. Nach $\mathrm{CH}_{\mathrm{H}}$. BelL (l. c., S. $146 \mathrm{ff}$.) wird diese Strafe mit Vorliebe bei Dieben und Einbrechern angewendet, was übrigens auch für den Kang zutrifft. Die Verurteilten dürfen sich ihren Lebensunterhalt bei Tag erbetteln. Nachts haben sie sich im Gefängnis einzufinden. In leichteren Fällen werden nur die Füße mit einer Kette verbunden, in schwereren durch eine Eisenstange ${ }^{18}$, die das Gehen noch mehr erschwert, oder es kommen die Handeisen hinzu oder der Kang und die Prügelstrafe. Mitunter werden auch zwei Verbrecher an den Füßen zusammengebunden. Die unter 4 genannte Strafe stammt wahrscheinlich aus China. Vgl. ąuch H. S. LANDOR (1. c., S. $410 \mathrm{ff}$.).

5. $r N a-m d a^{\prime}-g z e r-b a=D a s O h r$ mit einem Pfeil durchstoßen. Eine Strafe chinesischen Ursprungs, über deren Anwendung ich nichts ausfindig machen konnte.

6. rTa-lcag-gtong-ba $=$ Mit der Pferdepeitsche auspeitschen. Nach LANDOR (1. c., S. 396 mit Bild) liegt der Verurteilte von den Hüften an abwärts unbekleidet auf dem Boden. Während ihn eine Person hält, peitschen ihn zwei mit Lederriemen, in die manchmal Metallstücke eingeknotet sind. LANDORs Bericht entspricht genau unserem Bilde. Nach E. KaWAGUCHI werden die Lederpeitschen in Lha-sa durch frische Weidenruten ersetzt (1. c., S. 383) und nach A. TAFEI. in Osttibet durch Stöcke (1.c., S. 147). Die Zahl der Schläge kann sich nach Kawaguchi (1. c., S. 374) auf 300-700 erhöhen. Wird dabei das Opfer noch vor Beendigung der Strafe besinnungslos, so wird ihm Wasser gereicht, damit es sich für die Fortsetzung der Prozedur erholt. Mitunter endet die Strafe tödlich. Absichtliches Totpeitschen kommt nach P. LANDoN 19 in Lha-sa bei Ehebruch vor, wobei vorher den Verurteilten Nase und Ohren oder Nase und Lippen abgeschnitten werden. Auch Mord wird mit Totprügeln bestraft, dem schwere Foltern, wie Zertrümmerung der Gelenke, vorangehen. Im allgemeinen ist die Prügelstrafe mit den verschiedensten Strafen kombiniert. Nach $\mathrm{H}$. Harrer (1. c., S. 188) findet sie in Zentraltibet bei politischen Schädlingen, nach Cunningham .im Westtibet (1. c., S. 265) und nach A. Tafel in Osttibet (l. c., S. 147) besonders bei erstmaligem schweren Diebstahl Anwendung, vor allem, wenn der Dieb für die Rückerstattung des gestohlenen Gutes zu arm ist. Dem Geldwert des gestohlenen Gutes entsprechend wird die doppelte Zahl von Schlägen erteilt.

7. Lag-pa-bcod [gcod]-pa = Hände abhauen. Nach Cunningham (1.c., S. 263) wird bei Kindesmord in Westtibet in Verbindung mit Verbannung eine Hand abgehauen. Aus Ladak (tib.: La-dvags) berichtet S. H. RıввAсн (in: Drogpa Namgyal, München 1940, S. 214) von einem Fall, wo gegen Verletzung der Reinheitsvorschriften im Zusammenhang mit einem Sterbefall einer Frau eine Hand abgehauen wurde.

Bei zweitem Diebstahl schlägt man in Westtibet die linke Hand, bei drittem die rechte ab. Bei viertem Diebstahl wird der Verbrecher ertränkt (vgl. Bild 12, Cunningham, 1. c., S. 265). Nach Ch. Bell (1. c., S. 146) wird in Zentraltibet auch bei schwerwiegender Urkundenfälschung eine Hand abgehauen, in Osttibet nach A. TAFEL (1. c., S. 147) bei gewaltsamem Raub (Raubmord) oder, wie in Ladak, im Wiederholungsfalle von Diebstahl, wofür manchmal auch die Kniescheibe oder das zweite Auge entfernt wird. Bei erstem Diebstahl schneidet man nach Tafel in Osttibet die Nase oder ein Ohr ab,

18 A. Bollerup Sörensen, Ad Asiens ukendte veje, Kopenhagen 1951, Bd. II, Abb. 133.

19 Perceval Landon, Lhasa, London 1905, Bd. 2, S. 378. 
was in Zentraltibet auch bei besonders schweren Fällen von Ehebruch auf Wunsch des Betrogenen geschehen kann (E. Kawaguchi, 1. c., S. 384), oder es wird ein Auge ausgestochen. In Lha-sa, wo nach E. Kawaguchi (1. c., S. 384) die Hände erst nach dem fünften oder sechsten Diebstahl abgeschlagen werden, bindet man die Hände zwölf Stunden vor ihrer Entfernung so straff $a b$, daß sie völlig gelähmt sind. Die Stümpfe werden in kochendes Fett getaucht, um Verblutung zu verhindern.

8. Lag-pa-lko [ko]-tum [btum] = Hände in Tierhaut einnähen. Nach JäschKE (l. c., S. 5) handelt es sich in Zentraltibet um die Stümpfe der abgehauenen Hände. Nach Aussage von Einwohnern von Leh (Sle) stellt unser Bild aber eine viel grausamere Strafe als das Abhauen der Hände dar. Die nicht amputierten Hände werden in Lederbeutel eingenäht, die mit Salz gefüttert sind, das die Hände langsam zerfrißt.

9. Mig-mdon ['don]-pa =Blenden (tib. auch: Mig-bcar-ba). Die Form mdonpa für 'don-pa ist auch sonst noch zu belegen und dürfte darum kaum als Schreibfehler anzusehen sein. Das Entfernen der Augen durch ausstechen, ausreißen oder ausquetschen. In Osttibet wird nach A. TAFEL (1.c., S. 147, vgl. die Anmerkung zu Bild 7) bei Raub oder bei Diebstahl ein Auge entfernt, im Wiederholungsfalle auch noch das zweite. Nach E. Kawaguchi (1. c., S. 384) soll die Strafe des Blendens bei Diebstahl auch in Zentraltibet vorgekommen sein. Mit völliger Blendung wird ferner Raubmord bestraft. Nach $\mathrm{CH}_{H}$. BELL (l. c., S. 146) und H. Harrer (l. c., S. 188) werden schwere politische Verbrecher, insbesondere Revolutionäre und Attentäter geblendet. Bei Standespersonen wurde die Strafe des öftern durch das Überreichen einer schwarzen Binde angekündigt.

Mitunter wird die Prozedur Mig-'don-pa auch als Folter verwendet (J ÄschKE, l. c., S. 371).

10. Tshe-gang-brtson [btson]-'jug = Lebenslängliche Haft. Die Verurteilten müssen ihre Zeit im Gefängnis mitunter gefesselt verbringen. Die Nahrung ist kaum ausreichend, weswegen die Verwandten beisteuern müssen. Aber auch dann wird das meiste von den Wärtern (bTson-rdsi) verwendet (vgl. CunNinghaM, 1.c., S. 265). Viele politische Gefangene fristen in einsamen Gefängnissen (bTson-khang bzw. bTson-ra) ein elendes Dasein. So berichtete J. Cl. White ${ }^{20}$ von der gefangenen Witwe des verurteilten Staatsbeamten Nor-bu-tshe-ring, die mit ihrem Manne in eine Revolte gegen den XIII. DalaiLama verwickelt war und die E. Kawaguchi (1. c., S. 378) vor ihrer Verbannung in ein schauerliches Gefängnis unweit der bhutânesischen Grenze noch in Lha-sa im schweren Kang gesehen hat (vgl. die Anmerkung zu Bild 2).

11. $s K e-t u b[g t u b]-p a=K o ̈ p f e n$. Vgl. auch LANDor (1.c., S. 427 f.) und über die Anwendung dieser Strafe sowie ihre gelegentliche Umwandlung in Sklaverei, wobei vornehmlich religiöse Gründe mitsprechen, A. TAfEL (1. c., S. 147). Blutvergießen soll nach Möglichkeit vermieden werden, ein buddhistischer Grundsatz für den Strafvollzug. Nach Cunningham (1.c., S. 264) wird dementsprechend in Westtibet weitgehend verfahren, wovon lediglich das gelegentliche Abhauen der Hände abweicht. In Osttibet dagegen kommt die blutige Todesstrafe des Köpfens - wahrscheinlich unter chinesischem Einfluß -recht häufig vor. Wie schon oben angedeutet, führt nach tibetischer Vorstellung das Enthaupten zu Schwierigkeiten im Schattenreich des Jenseits. Eine Neugeburt zur Besserung des Karma ist für den Enthaupteten ausgeschlossen. Darum werden auch andere Todesstrafen dadurch verschärft, daß man dem

20 J. Cl. Whiтe, Sikhim and Bhutan, London 1909, S. 204. 
Leichnam den Kopf abschneidet (vgl. die Anm. zu Bild 12), was mit «Ewige Verdammnis » bezeichnet wird.

12. g.Yar-rkyur [skyur]-churkyur $[$ skyur $]=$ Hinabstürzen (bzw.) in den Flu $\beta$ hinabstürzen. Diese Todesstrafe wird der blutigen Enthauptung aus den bereits angegebenen Gründen vorgezogen. Hände und $F \ddot{u}-$ Be werden gebunden. Der $\mathrm{Hals}$ wird mit einem Gewicht beschwert. Manchmal wird dem Leichnam des Ertränkten der Kopf abgeschnitten (E. KawAGUCHI, l. c., S. 385), was als Strafverschärfung angesehen wird (vgl. die Anmerkung zu Bild 11). Nach JÄschke (1. c., Seite 28) handelt es sich bei dem Ertränken in der angegebenen Weise ursprünglich um eine
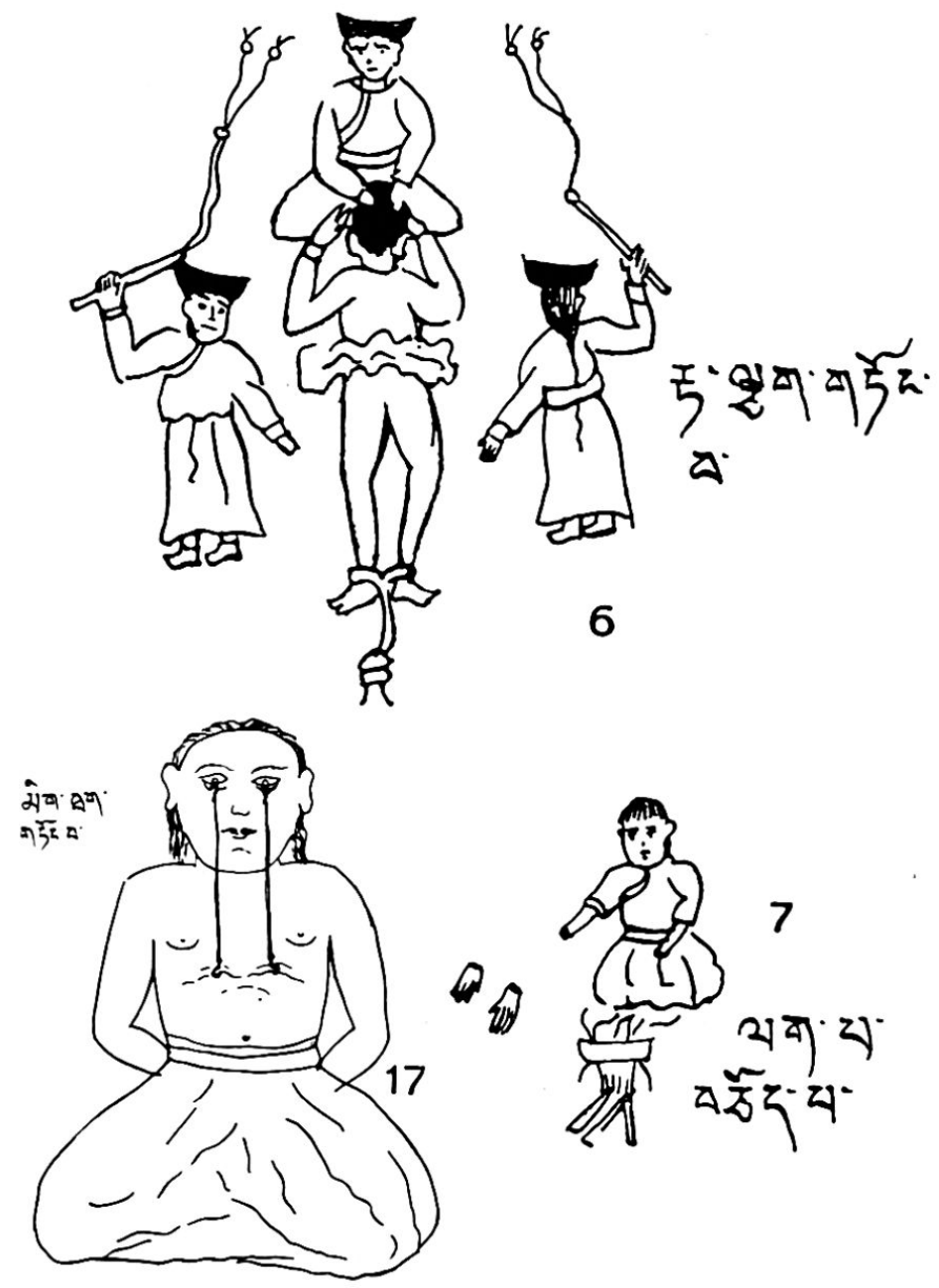
zentraltibetische Strafe, worauf auch unser Bild Bezug nimmt, indem der Felsen als 1Chag [1Chags]po-ri bezeichnet wird, d. h. als jener steile Berg bei Lha-sa, auf dem sich die berühmte medizinische Klosterakademie befindet (auf unserer Zeichnung mit 1 Ha-khang bezeichnet). Durch Cunningham (l.c., S. 263) ist diese Strafe auch für Westtibet bezeugt. Dort werden die Verurteilten von Felsvorsprüngen oder Brücken in die Flüsse gestürzt. Als Verfehlung der Verurteilten nennt Cunningham Mord, vierten Diebstahl (vgl. Anm.zu Bild 7) und Tempelraub. Bei Totschlag im Verlauf einer Rauferei wurde in Zentraltibet der Totschläger an den Leichnam gebunden und nach 24 Stunden mit diesem zusammen in den Fluß geworfen (Cunningham, l. c., S. 263). Diese Strafe konnte auch in Haft, Geld- oder Prügelstrafe verwandelt werden, da eben die Lebensberaubung der buddhistischen Religion prinzipiell entgegensteht.

Einen besonders tragischen Fall einer Hinrichtung durch Ertränken berichten ausführlich E. KAwAGUCHI (1. c., S. 16 ff. mit Abb. S. 18) und Perceval Landon (1. c., S. $235 \mathrm{ff}$.). Dabei handelt es sich um den gelehrten und edlen Lama Seng-ge-chen-rdo-rje-'chang, dessen Schuld darin gesehen wurde, daß er Sarat Chandra Das ${ }^{21}$ auf seiner Forschungsreise im Jahre 1882 unterstützt hatte. Der Lama wurde als politischer und religiöser Verbrecher hingerichtet, indem er nicht von einem Felsvorsprung hinabgeworfen, sondern mit Hilfe eines durch Steine beschwerten Seiles ins Wasser versenkt und nach etwa 10 Minuten wieder herausgezogen wurde. Diese grausame Prozedur mußte mehrmals wiederholt werden, bis der Verurteilte ertrunken war.

21 s. Anm. 4. - Vgl. auch D. Macdonald, Twenty Years in Tibet, London 1932, S. $137 \mathrm{f}$. 
Für Tempelraub gibt Cunningham (1.c., S. 264) neben Ertränken auch die Kreuzigung an. Der Delinquent wird mit Stricken an ein Andreaskreuz gebunden, das an einem Pfahl befestigt ist. Er wird dann entweder dem Tod durch Verhungern und Verdursten preisgegeben oder er wird, wir F. DE FILIPPI 22 berichtet, durch kochendes Öl oder durch Beschuß mit Pfeilen zu Tode gequält ${ }^{23}$.

13. $1 K_{0}[\mathrm{Ko}]$-tum [btum]-rgyab-pa $=$ In Tierhaut eingenäht werden. Der Verurteilte wird in den Fluß geworfen. Diese Strafe soll aus Zentraltibet stammen

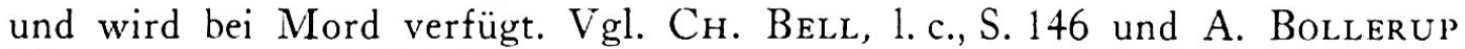
Sörensen, l. c., S. 324 f.

Für die folgenden Strafen war es mir nicht möglich, ihre Anwendung ausfindig zu machen:

14. Ka-cig [gcig]-sgo-cig [gcig] = Kleines Haus (vgl. Jäschke, 1. c., S. 1). Der Verurteilte wurde in einem Kerker an eine Säule gebunden und dem Hungertod überlassen.

15. $r$ Gyug-brtsi [brtsir]-gtong-ba $=$ Gewaltsam zerquetschen. Die kleinen Punkte auf der Zeichnung sollen das Blut des Gemarterten darstellen.

16. bSal [gSal]-shing-la-skyon-pa = Auf spitzem Pfahl aufspießen. Über die Pfählung vgl. auch A. Grünwedel, Die Legenden des Nâ-ro-pa, Leipzig 1933, S. 51, 14 A: Srog-'dor-shing-sa-steng-btsugs-nas (= «... einen Pfahl, auf dem man das Leben verliert, in die Erde gerammt...»). Vgl. ferner A. GrünweDEL, Die Teufel des Avesta, Berlin 1924, Bd. 2, S. 46-49).

Auf unseren Bildern nicht angegeben ist die schwerste aller Verstümmelungsstrafen (gCod-'breg-gi-khrims ${ }^{24}$ ), zu denen ja auch das Enthaupten gerechnet wird, und neben dem Pfählen die grausamste 'Todesstrafe überhaupt. Bei ihr wird der Verurteilte langsam in Stücke geschnitten. Für Tibet ist diese qualvolle Hinrichtung chinesischen Ursprungs durch Perceval Landon (l. c., S. 378) bezeugt. Das Opfer wird an einen Pfahl gebunden. Durch acht bis sechsunddreißig (früher bis zu hundertundzwanzig) Schnitte werden immer mehr Körperteile entfernt, wobei der Tod möglichst erst am Ende der festgelegten Prozedur eintritt ${ }^{25}$. In China wurden noch im 20. Jh. in den letzten Jahren der Mandschudynastie Landesverräter, Vater- und Mutter- und weibliche Gattenmörder öffentlich auf diese Weise bestraft, was durch eine Fülle von Photomaterial dokumentiert werden kann. Der von Perceval LanDON für Tibet bezeugte Fall bezog sich auf Landesverrat.

Im folgenden gebe ich eine Übersicht der Vergehen und der Strafen, die im Zusammenhang mit der Besprechung unserer Bilder erwähnt wurden. Daß die Bestrafung in den verschiedenen Landesteilen große Abweichungen zeigt, muß nochmals unterstrichen werden. In der Praxis werden auch manche Strafen kombiniert, so z. B. Kang, Hand- und Fußeisen und die Prügelstrafe. Gerade das Verprügeln ist, wie sich gezeigt hat, mit den verschiedensten Strafen verbunden, wobei nach der ortsüblichen Praxis oder nach dem Gutdünken des Richters verfahren wird. Unsere Aufstellung kann also nur einen höchst unbestimmten Eindruck vom tibetischen Strafverfahren vermitteln.

${ }^{22}$ F. DE FiLippi, An account of Tibet, 2. Aufl. London 1937, S. 174. - Vgl. Cunningham, 1. c., S. 264 .

${ }^{23}$ Ein Photo einer solchen Hinrichtung in Kaschgar bringt G. DE RœRICH, Sur les Pistes de l'Asie Centrale, Paris 1933, Tafel XIII.

24 Sarat Chandra Das, 1. c., S. 390.

25 Vgl. auch B. Navarra, China und die Chinesen, Bremen 1901, Bd. 2, S. 111. Chinesisch heißt diese grausame Strafe Ling-Ch’ih (wörtlich: Langsame Folter). 
Diebstahl und Einbruch: Kang (Zentraltibet, 1 und 2).

Stock (Zentraltibet, 3).

Fuß- und Handeisen (Zentraltibet, 4).

Die genannten Strafen meist kombiniert (Zentraltibet ${ }^{26}$ ).

In schwereren Fällen :

(zum 1. Mal gegriffen) Prügelstrafe, oft in Verbindung mit Kang, Stock oder Hand- bzw. Fußeisen (Zentraltibet, 4).

In Verbindung mit Vermögenskonfiskation (West- und Osttibet, 6).

Nase oder ein Ohr abschneiden (Osttibet, 7).

Ein Auge entfernen (besonders bei Raub und Viehdiebstahl) (Osttibet, 9).

(zum 2. Mal gegriffen) Linke Hand abschlagen (bes. bei Viehdiebstahl) (West- und Osttibet, 7).

Kniescheiben ausschneiden (Osttibet, 7).

Entfernen des zweiten Auges (Osttibet, 7 und 9)

(zum 3. Mal gegriffen) Rechte Hand abschlagen (Westtibet, 7).

(zum 4. Mal gegriffen) Ertränken (Westtibet, 12).

(zum 5. Mal gegriffen) Beide Hände abhauen (Zentraltibet, 7).

Ehebruch :

Die Nase und beide Ohren abschneiden (Zentraltibet, 6).

Nase und beide Ohren oder Nase und Lippen abschneiden mit folgendem Totpeitschen (Zentraltibet, $6^{27}$ ).

Mord :

Verbannung mit Brandmal, oft mit vorhergehender Prügelstrafe (Westtibet, 2). Ertränken (Westtibet, 12; Zentraltibet, 13).

Köpfen (Osttibet, 11).

Zertrümmerung der Gelenke mit einem Hammer und anschließendes Totpeitschen (Westtibet und westl. Zentraltibet, $6^{28}$ ).

Raubmord: $\quad$ Kniescheiben ausschneiden (Osttibet, 7).

Eine Hand abschneiden (Osttibet, 7).

Ausstechen beider Augen (Osttibet, 9).

Köpfen (Osttibet, 11).

Kindesmord: $\quad$ Eine Hand abhauen mit folgender Verbannung (Westtibet, 7).

Totschlag :

Ertränken; der Verurteilte wird mit dem Leichnam des Erschlagenen im Wasser versenkt (Westtibet, 12).

Politische Vergehen je nach Schwere der Schuld:

Kang (Zentraltibet, 1 und 2).

Prügelstrafe (Zentraltibet, 6). Meist geht die Prügelstrafe den übrigen Strafen für politische Verbrechen voran ${ }^{28}$.

Kang mit folgender Verbannung oder mit lebenslänglichem Gefängnis (unter Umständen auch in der Verbannung) (Zentraltibet, 2 und 10).

Blenden beider Augen (Zentraltibet, 9); insbesondere bei Attentatsversuchen belegt.

Ertränken (Zentraltibet, 12 und 13).

Todesstrafe in Form des Durchstoßens der Schläfen mit glühenden Eisenstäben ${ }^{28}$.

Landesverrat: $\quad$ Langsam in Stücke zerschneiden (Zentraltibet).

Tempelschändung und Tempelraub: Ertränken (Westtibet, 12).

Kreuzigung (Westtibet, 12).

Verletzung der Reinheitsgebole: Eine Hand abhauen (Westtibet, 7).

Der Strafvollzug erfolgt im allgemeinen sofort auf die Urteilsverkündung. Nachdem der Kläger seine Angelegenheit dem Gemeindevorsteher ('Go-pa) seines Dorfes oder dem Bürgermeister (bLon-po) seiner Stadt angezeigt hat, wird dem Distrikts-

${ }^{26}$ Nach W. Fitchner (Om mani padme hum, 8. Aufl. Leipzig 1930, S. 222) werden die zum Kang oder zu Hand- und Fußeisen oder zu beidem Verurteilten nachts im Gefängnis in den Stock gelegt. Im Gefängnis von Nag-chu-kha sah der Forscher Vierkantbalken ähnlich unserer Abb. 3 mit achtzehn Doppellöchern für die Hand- und Fußgelenke. Das Photo eines Mannes mit rundem Kang und mit Fußeisen zeigt FII.CHNER in dem genannten Buche auf S. 240.

${ }^{27}$ Im allgemeinen kommen diese Strafen kaum zur Anwendung, da Ehebruch meist nicht sonderlich tragisch genommen wird. Dafür werden, wenn auf Ehescheidung bestanden wird, vermögensrechtliche Auseinandersetzungen vorgenommen (vgl. Сн. BeLl, The People of Tibet, Oxford 1928, S. 195).

28 Swami Pranavânanda, Kailâs-Mânasarôvar, Calcutta 1949, S. 76 und zugehörige Abb. 41 mit Folter- und Strafwerkzeugen. 
vorsteher (bKa'-blon) Bericht erstattet und die Gerichtsversammlung einberufen. Letzteres erfolgt in den größeren Städten durch den damit beauftragten zuständigen obersten Richter (gShags-dpon). Die Gerichtsversammlung besteht aus fünf oder auch sieben Ältesten (rGad-pa) und zwei oder auch mehr mit dem landesüblichen Recht (Yul-khrims) vertrauten Rechtssachverständigen (Khrims-dpon). Die Versammlung findet im Gerichtsgebäude (gShags-khang) statt und wird durch das Blasen der Gerichtstrompete (Khrims-dung) eröffnet und beendet ${ }^{29}$.

29 Vgl. A. Cunningham, 1. c., S. 262.

\title{
PEINES ET TORTURES CHEZ LES THIBÉTAINS
}

La bibliographie spécialisée ne comprend jusqu'ici que quelques indications sur la justice thibétaine. Les deux planches (non publiés jusqu'à maintenant), dessinées par un 'Thibétain, montrent les peines corporelles les plus courantes. Des différences dans l'application et importance des peines se font jour particulièrement entre l'ouest et l'est du Thibet. Une réglementation unifiée manque, les peines varient fortement d'un endroit à l'autre. On perçoit, à côté d'anciennes traditions nomades, des influences chinoises. Parmi les peines capitales, les mutilations du corps ainsi que la décapitation semblent être les plus fortes, car leurs effets se font sentir jusque dans l'au-delà. Le treizième dalaï-lama (1933) tenta d'introduire un adoucissement à cet état moyenâgeux. Avec l'incorporation du Thibet dans la République populaire de Chine, la justice thibétaine et l'application des peines changera assurément de manière fondamentale.

\section{DIE ORTSGEBUNDENHEIT DER FRAU BEI DEN MOZABITEN}

\author{
KARL SUter 1
}

Die Mozabiten bewohnen den als Mzab bekannten Abschnitt der nordalgerischen Sahara. Sie sind Berber, sprechen einen eigenen berberischen Dialekt und gehören in religiöser Hinsicht zu den Ibaditen, einem $Z_{\text {weig der }}$ Kharidjiten ${ }^{2}$. Ihr glühendes Bekenntnis zum gemeinsamen Glauben schließt sie zu einer engen religiös-sozialen und politischen Gemeinschaft zusammen, die eifersüchtig auf ihre kulturelle Selbständigkeit bedacht ist.

Um den religiösen Verfolgungen von seiten der Anhänger anderer muselmanischer Glaubensrichtungen zu entgehen, flüchteten sie in die Einsamkeit der Sahara und gründeten im Laufe des 11. Jahrhunderts im Tal des Oued Mzab fünf nahe beieinanderliegende Oasen, nämlich Ghardaia, den Hauptort (1954 15 69o Einwohner), dann Melika, Ben Isguen, Bou Noura und El Ateuf. Dazu kamen im 17. Jahrhundert die beiden abseits gelegenen Oasen Berrian und Guerrara. Bei all diesen Gründungen handelt es sich um Ksour (Einzahl Ksar), d. h. um geschlossene, von turmbewehrten Ringmauern umgebene, stadtähnliche Siedlungen. In ihnen wohnen, das Arabernest Metlili miteinbezogen, zusammen 52641 (1954) Einwohner. Von diesen sind ungefähr 40000 Mozabiten, 11000 Araber und 1200 Juden. Diese Bevölkerung lebt hauptsächlich vom Gartenbau, im besondern vom Unterhalt der Dattelpalme. Der außerordentlich karge Boden vermag sie aber nicht zu ernähren. Darum arbeitet ein großer Teil der erwachsenen Mozabiten - ihre Zahl wird auf 9000 geschätzt - auswärts in den größeren Ortschaften Algeriens, namentlich in Algier, Constantine und Oran. Die Auswanderer, die ihre Familien in der Sahara zurücklassen, kehren aber von Zeit zu Zeit ins Mzab zurück.

An der Spitze der mozabitischen Religionsgemeinschaft steht in jeder ihrer sieben Siedlungen die Halga («Kreis»), die sich aus 13 Schriftgelehrten, den Azzaba (mozabitisch; arabisch Tolba) zusammensetzt. Der Fähigste oder Frömmste unter ihnen amtet, durch die Halga auf Lebenszeit dazu gewählt, als Scheich. Dieses Kollegium besorgt alle Obliegenheiten der Moschee, verwaltet deren Güter, ruft die Gläubigen zu den gemeinsamen Gebeten zusammen, bestreitet den Koranunterricht an die Knaben und überwacht das durch strenge Sittengesetze geregelte religiös-sittliche Leben der ganzen Gemeinschaft. Rauchen und Alkoholgenuß

1 Forschungsreise 1955, in verdankenswerter Weise subventioniert vom Schweizerischen Nationalfonds.

${ }^{2}$ Ortsnamen und auch eine Anzahl Begriffe werden in französischer Schreibweise wiedergegeben. 